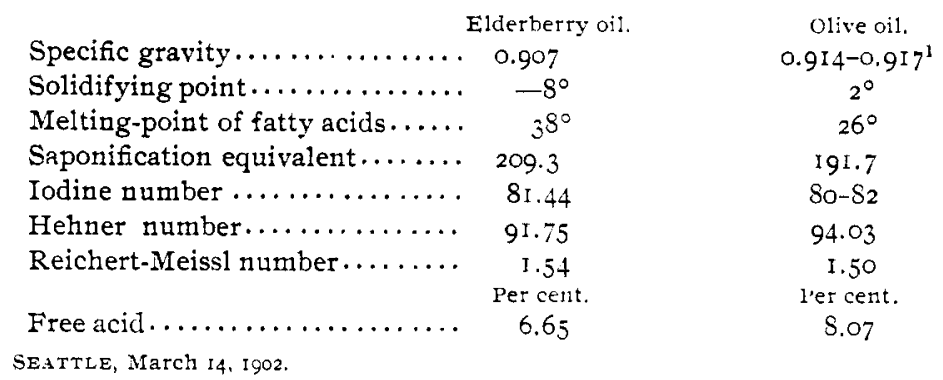

\title{
A VOLUMETRIC METHOD FOR THE ESTIMATION OF SUL- PHURIC ACID IN SOLUBLE SULPHATES.
}

By Yastjuro Nikaido.

Received September 12, Iqor.

$\mathrm{THF}_{\mathrm{F}}$ method is based on the following reactions:

(I) $\mathrm{K}_{2} \mathrm{SO}_{4}+\mathrm{Pb}\left(\mathrm{NO}_{3}\right)_{2}=\mathrm{PbSO}_{4}+2 \mathrm{KNO}_{3}$.

(2) $2 \mathrm{KI}+\mathrm{Pb}\left(\mathrm{NO}_{3}\right)_{2}=\mathrm{PbI}_{2}+2 \mathrm{KNO}_{3}$.

Under proper conditions it was found that lead salts will not react with iodides in the presence of sulphates until all the sulphuric acid has been precipitated by the lead, whereupon the yellow color of the lead iodide becomes visible. It is, therefore, possible to use potassium iodide as an indicator for the end reaction between lead and sulphuric acid.

To test the accuracy of the method, N/Io solutions of lead nitrate and of potassium sulphate were used. Then Io $\mathrm{cc}$. of the potassium sulphate solution were taken, about 0.2 gram of potassium iodide added and the solution was titrated with a lead nitrate solution. It was found that on adding lead nitrate, lead iodide was first formed, imparting a yellow color to the solution. This color quickly disappears, owing to the formation of lead sulphate. As soon as all the sulphuric acid is precipitated the yellow color becomes permanent. In water solutions alone, however, the reaction is not complete, lead sulphate being somewhat soluble in water and the yellow color of the lead iodide showing slightly, even when an excess of potassium sulphate is present.

To avoid this, it was determined to try the reaction in an alco1 Meyer and Jacobson: "Lehrbuch," vol. I, p. 594. 
holic solution, and for this purpose another Io $c c$. of the potassium sulphate solution were taken together with about 0.2 gram of potassium iodide. Alcohol was then added until a slight precipitate of potassium sulphate was formed, and then the lead nitrate solution was carefully added. It was found that the color of lead iodide appeared just about when Io cc. of lead nitrate solution had been added.

Experiments were made to find out in what strength of alcohol the reaction best takes place. Since in water alone it is impossible to obtain any satisfactory results, 25 per cent. alcohol was first used. With this, it was also impossible to obtain good results. Then a titration was made using 35 per cent. alcohol, but still the results were not satisfactory. In 40 per cent. alcohol the results were fair, but the reaction took place very slowly. In 50 per cent. alcohol, the results were fairly satisfactory, although the reaction was still slow. The results obtained from the titrations in 60 per cent. alcohol were satisfactory, and the reaction was moderately rapid. The titrations in 65 per cent. alcohol gave very satisfactory results, and the reaction went quite rapidly with the following results :

\begin{tabular}{|c|c|}
\hline $\begin{array}{l}\text { N/Io } \\
\text { potassium sulphate } \\
\text { solution. } \\
\text { cc. }\end{array}$ & $\begin{array}{l}\text { N/Io } \\
\text { lead nitrate } \\
\text { solution. } \\
\text { cc. }\end{array}$ \\
\hline 5.00 & 5.02 \\
\hline 10.00 & 10.05 \\
\hline II.25 & II .22 \\
\hline 19.97 & 20.01 \\
\hline Average 1.00 & 1.0017 \\
\hline
\end{tabular}

The results from the titrations in 70 per cent. alcohol were obtained in the same manner as in the 65 per cent. alcohol. It was found at this point, viz., with 70 per cent. alcohol, or over, the reaction began to go more slowly and to become increasingly diffcult to complete, owing to the insolubility of potassium sulphate in alcohol. The experiments show therefore that the reaction goes best in alcoholic solutions from 50 to 70 per cent.

Experiments were made to find out what effect various acids have upon the reaction. It was found that no satisfactory results could be obtained in the presence of free nitric acid, since it liberates iodine from potassium iodide and makes it difficult to see the color of the end reaction. In the presence of hydrochloric acid, 
the reaction does not take place normally, because lead iodide is converted into a pale yellow compound (probably a double halogen salt of lead) which is not decomposed by neutral sulphates. The same compound is formed when a large quantity of chlorides is present. Small quantities of chlorides do not affect the titration. In the presence of free acetic acid the reaction takes place regularly and satisfactorily, but sodium acetate interferes with the titration, owing to the very considerable solubility of lead iodide in sodium acetate. The titrations were made also in the presence of free oxalic and tartaric acids. In the oxalic acid solution lead was precipitated, but not in the tartaric acid solution. In presence of potassium tartrate, lead was precipitated.

To determine the applicability of this method to stronger solutions of sulphates, a $\mathrm{N} / 2$ solution ( 43.47 grams per liter) of potassium sulphate was used. Portions of this solution were titrated with an $\mathrm{N} / 2$ solution ( 82.5425 grams per liter) of lead nitrate. It was found that in this case the titration was most satisfactory, using 50 per cent. to 60 per cent. alcohol.

The results were as follows:

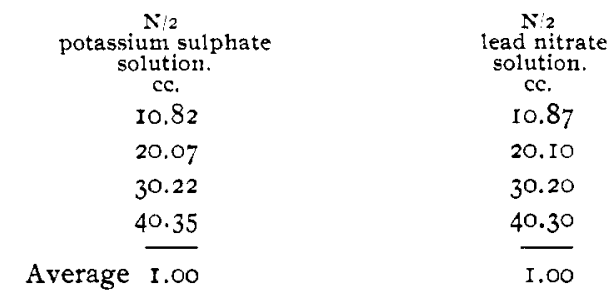

Having in the manner indicated determined the accuracy of the method under the conditions mentioned, its applicability for the estimation of sulphuric acid in other sulphates was next tried. The substances used were magnesium sulphate, zinc sulphate, ferrous ammonium sulphate, copper sulphate, manganous sulphate, ferrous sulphate, and ferric ammonium sulphate.

In the titration with ferrous ammonium sulphate a few drops of acetic acid were added to prevent the formation of insoluble basic compounds. In the case of copper sulphate the copper was precipitated with zinc or aluminum and the titration carried out as usual after acidifying the solution with acetic acid in order to prevent the formation of precipitates of basic sulphates.

In the case of ferric ammonium sulphate, the iron was precipi- 
tated with potassium hydroxide. The solution and precipitate were then transferred to a graduated flask and made up to a definite volume. The precipitate was allowed to settle and an aliquot part of the supernatant solution was taken and acidified with nitric acid, evaporated to dryness to expel free nitric acid, dissolved in water, alcohol added, and the solution titrated with lead nitrate. The error due to the volume of the ferric hydroxide is negligible in an $\mathrm{N} / \mathrm{ro}$ solution in comparison with the errors of titration. In these determinations, pure substances which were obtained by recrystallization, and with each, semi-normal solutions were used. The results in all these titrations were fair and satisfactory.

The following precautions should be carefully observed. The titrations should never be made in warm solutions as lead iodide is soluble in hot water. The titrations should not be made in a large volume of solution as lead iodide is slightly soluble even in cold water.

It is probable that the method is applicable to all soluble sulphates, provided:

(I) That the operation is carried out in dilute alcohol, that is, 50-70 per cent. alcohol in case a tenth-normal solution of lead nitrate is used, and 50-60 per cent. alcohol in case a semi-normal solution of lead nitrate is used.

(2) That nitric acid, hydrochloric acid, sodium acetate and substances that will form insoluble compounds with lead are avoided.

(3) That in the case of colored sulphates, the base which gives color to the compound is removed by precipitation, e. g., in the case of copper sulphate, the copper is precipitated, using zinc or aluminum; in the case of ferric ammonium sulphate, the iron is precipitated with potassium hydroxide.

(4) That in the case of the sulphates of the heavy metals, such as ferrous sulphate, ferrous ammonium sulphate, zinc sulphate, etc., a few drops of acetic acid are added to prevent the formation of basic sulphates.

(5) That the titrations are made in small volumes of cold solution, using about 0.2 gram of the potassium iodide to each $25 \mathrm{cc}$. of the solution.

Finally, it may be claimed for the method here proposed, that its 
accuracy, rapidity, ease of manipulation and general applicability should render it more satisfactory than any of the previously known volumetric methods for the estimation of sulphuric acid.

The method may also be used for the estimation of lead in soluble compounds of lead by reversing the operation.

\title{
A PRELIMINARY NOTE ON THE OIL OF MILFOIL.
}

\author{
BY A. B. ALBERT.
}

Received April 30, 1902.

THE specimen examined was distilled by Metzner and Otto, of Leipzig. It is of a dark bluish green color and has a mild and pleasant odor. Specific gravity at $22^{\circ}$ equals 0.9217 . Refractive index, 1.506 at $20^{\circ}$. It is soluble in absolute alcohol, ether, xylene and chloroform; also in about 6.5 volumes of 95 per cent. alcohol with a slight residue of brownish colored oil.

It was distilled under reduced pressure with the following results :

One hundred volumes of the oil yielded:

7 parts by volume between $170^{\circ}-190^{\circ}$.

I7 parts by volume between $190^{\circ}-210^{\circ}$ (sp. gr. 0.900 ).

50 parts by volume between $210^{\circ}-220^{\circ}$ (sp. gr. 0.907 ).

9 parts by volume between $220^{\circ}-235^{\circ}$ (sp. gr. 0.936 ).

3 parts by volume above $235^{\circ}$.

The residue, constituting I4 parts, remaining in the flask was a semi-solid, dark olive-brown, waxy substance, hardly soluble in cold alcohol, partly soluble in boiling alcohol, yielding a dark yellow-brown solution. The insoluble residue was of a greenish brown color, somewhat resembling paraffin in consistency. By evaporation of the alcoholic solution a dark yellow-brown pitch was obtained.

The oil has a slightly acid reaction.

The products of distillation gave the following reactions:

$170^{\circ}-190^{\circ}$, slightly acid.

$190^{\circ}-210^{\circ}$, nearly neutral.

$210^{\circ}-220^{\circ}$, nearly neutral.

$220^{\circ}-235^{\circ}$, slightly acid.

(This distillate contained products of decomposition.)

That portion distilling between $170^{\circ}-190^{\circ}$ was tested ror cineol, 